\title{
18F-FDG PET/CT for first follow-up post chemotherapy in lymphoma: Is it mandatory to do whole body scan?
}

\author{
Hussein Farghaly1,2*, Hatem Nasr²,3, Abdullah Al Qarni² and Wala Elhussein ${ }^{2}$ \\ 1 Nuclear Medicine Unit, Department of Clinical Oncology, Assiut University, Assiut, Egypt \\ ${ }^{2}$ Department of Radiology, Prince Sultan Military Medical City, Riyadh, Saudi Arabia \\ ${ }^{3}$ Nuclear Medicine, Kasr El Einy Hospital, Cairo University, Cairo, Egypt
}

\begin{abstract}
Objective: To assess the feasibility of 18F-2-fluoro-2-deoxy-D-glucose positron emission tomography/computed tomography (FDG-PET/CT) scan limited to the original sites of lymphoma in $1^{\text {st }}$ follow-up (F/U) post-chemotherapy to reduce patient's radiation exposure and scan time. Patients and methods: FDG PET/CT scans of 100 lymphoma patients were reviewed and the sites of disease in pre-Chemotherapy and the $1^{\text {st }} \mathrm{F} / \mathrm{U}$ post-chemotherapy scans were recorded. The supposed saved time from PET part of the scan and reduction in radiation dose from CT part if PET/CT scan limited to the original sites of lymphoma in $1^{\text {st }} \mathrm{F} / \mathrm{U}$ post-chemotherapy scan is used, were calculated. Result: $45 \%$ of $1^{\text {st }}$ F/U post-chemotherapy PET/CT scans showed no significant residual FDG-uptake indicating complete metabolic response. Significant residual FDG-uptake at known disease sites was seen in $55 \%$ of $1^{\text {st }} \mathrm{F} / \mathrm{U}$ post-chemotherapy scans indicating residual disease. No lesions with significant FDG uptake in new sites were detected in the $1^{\text {st }} \mathrm{F} / \mathrm{U}$ post-chemotherapy PET/CT indicating no unexpected sites of lymphoma. The supposed reduction in the mean scan time and radiation dose is $5.3 \pm 1.47$ Min and $4.2 \pm 1.2$ mSv respectively if scan limited to the sites of known disease is used in $1^{\text {st }} \mathrm{FU}-\mathrm{P}-\mathrm{CHT}$, without missing any significant findings. Conclusion: It is not mandatory to do WB 18F-FDG PET/CT scan in assessment of early response to chemotherapy in curable lymphoma and it may be sufficient to limit scans to the sites of known disease without missing any significant findings, resulting in significant reduction in total radiation dose and time saving.
\end{abstract}

Keywords: post-chemotherapy; FDG PET/CT; lymphoma; radiation dose; chemotherapy

\section{Introduction}

18F-FDG PET/CT is a cornerstone procedure nowadays in lymphoma management used as established standard in the initial staging, monitoring the response to the therapy, and restaging after treatment of patients with Hodgkin's lymphoma ( $\mathrm{HL})$ and high-grade non- Hodgkin's lymphoma (NHL). Evaluation of response to chemotherapy is an important concern for the management of lymphoma patients. Separation of low-risk patients, who will be cured with conventional therapy and less intensive and less toxic regimens, from high-risk patients, for whom an early switch to alternative, more aggressive treatment strategies could increase the possibility of remission and cure, can be potentially achieved by early prediction of response to therapy. This is known as risk-adapted therapy [1]. Clinical, histopathological, and imaging criteria are used for assessment of response to chemotherapy [2].

For HL and aggressive NHL lymphoma, 18F-FDG PET/CT is normally used for post-treatment assessment as well as after as few as two cycles of chemotherapy as an interim scan [3]. The use of near simultaneous acquisition of PET and $\mathrm{CT}$ has resolved the limited spatial resolution of PET and has improved accurate anatomical localization of sites of radiotracer uptake detected on PET. However, this lead to increased radiation dose to the patient compared to either PET or CT alone. The radiation dose from PET/ $\mathrm{CT}$ results both from the injected radiotracer 18F-FDG and from the external dose of the CT component. Based on the effective dose of $0.019 \mathrm{mSv} / \mathrm{MBq}$ [0.070 rem/mCi] for FDG, Approximately $7 \mathrm{mSv}$ comes from an injected dose of $10 \mathrm{mCi}$ FDG [4]. The external dose of the CT component, which can run as high as $25 \mathrm{mSv}$, depending on the type of study performed as well as the anatomical region and number of body parts imaged [5]. Several recent studies

*Corresponding author: Hussein R. Farghaly, Department of Clinical Oncology and Nuclear Medicine, Assiut university, 71515 Assiut, Egypt. Tel.: 00966565964294; Fax: 00966114767348; Email: hussen2h@yahoo.com

Received 13 January 2015 Revised 5 March 2015 Accepted 15 March 2015 Published 22 March 2015

Citation: Farghaly $\mathrm{H}$, Nasr H, Qarni AA, Elhussein W. 18F-FDG PET/CT for first follow-up post chemotherapy in lymphoma: Is it mandatory to do whole body scan?. J Cancer Res Ther. 2015; 3(3):20-25. DOI: 10.14312/20524994.2015-4

Copyright: (c) 2015 Farghaly H, et al. Published by NobleResearch Publishers. This is an open-access article distributed under the terms of the Creative Commons Attribution License, which permits unrestricted use, distribution and reproduction in any medium, provided the original author and source are credited. 
have reported a typical average dose of $\sim 14 \mathrm{mSv}$ for skull base to thigh FDG-PET/CT examinations [6-10].

Currently there is a strong concern over radiation dose to patients, especially hybrid modalities due to their dual imaging nature. The two components of the PET/CT scan both impose a certain radiation burden. Although both modalities must maximize the diagnostic information obtained there is a strong trend to reduce the radiation dose. Many methods are currently available to reduce the radiation dose from $P E T / C T$. Reduction of the radiation dose from FDG injection can be achieved by newer imaging units with faster crystals, time-of-flight (TOF) [11, 12], and 3D PET emission acquisition mode which allows for a reduction in injected dose by up to $50 \%$ relative to the $2 \mathrm{D}$ mode [13]. Reduction of dose from the CT component of PET/CT may be undertaken by newer reconstruction techniques such as adaptive statistical iterative reconstruction (ASIR), modelbased iterative reconstruction (MBIR) and automatic tube current modulation which reduce patient dose exposure by $20-60 \%$ [14]. Also reduction of radiation dose from the CT component of PET/CT can be achieved by the reduction of the anatomical extent of attenuation-correction CT scan in certain situations as limited above diaphragm PET/CT for head and neck squamous cell carcinoma $[15,16]$ and limited pelvic PET/CT for suspected hip prosthesis infection [17].

In this study we investigate the feasibility to reduce scan time and radiation dose by using limited field of view early post therapy FDG PET/CT scan in lymphoma patients with imaging confined only to the region of the initial disease site as detected in base line scan.

\section{Materials and methods}

\section{Patient population}

100 lymphoma patients were involved in this study with the following inclusion and exclusion criteria: (a) Inclusion criteria: i. All patients should have baseline and early post chemotherapy FDG PET/CT scans, ii. All patients should have significant FDG avid disease in baseline FDG PET/ CT scans, iii. All patients should have at least one body region out of four regions (head and neck, chest, abdomen or pelvis) spared from disease involvement. (b) Exclusion criteria: i. Any patients with missed baseline or early post chemotherapy FDG PET/CT scans, ii. Any patients with extensive lymphomatous disease involved all the four body regions, iii. Any patients with low FDG avid disease in baseline FDG PET/CT scans.

\section{PET/CT scanning}

Patients fasted at least $4 \mathrm{~h}$ before the tracer injection and received an intravenous injection (some patients were injected manually and the others by automatic injector) of approximately $5.18 \mathrm{MBq} / \mathrm{Kg}(0.14 \mathrm{mCi} / \mathrm{Kg})$ of $18 \mathrm{~F}-\mathrm{FDG}$, with a maximum of $444 \mathrm{MBq}(12 \mathrm{mCi})$. Blood glucose level was measured immediately prior to FDG injection and was $<165 \mathrm{mg}$ in all studied cases. Patients were sitting calm in a quiet injection room without talking during the subsequent 40-60 min of the FDG uptake phase. Patients were allowed to breathe normally during image acquisition without specific instructions. All scans were acquired using a Gemini TF PET/CT scanner (Philips Medical Systems). Emission scans were acquired at 1 Min per bed position always in 3D which may increase up to 2 or 3 Min per bed position in case of obese patients depending on the body mass index (BMI). The FOV was from the base of the skull to mid-thigh with the arms above the head unless the patient cannot tolerate positioning the arm above the head, arms down position were used. The CT scans were used for attenuation correction purposes and to help in anatomic localization of FDG. The 3-dimentional (3D) WB acquisition parameters consisted of a $128 \times 128$ matrix and an $18 \mathrm{~cm}$ FOV with a $50 \%$ overlap.

\section{CT scanning}

The CT scan of the PET/CT scanner consisted of a 16 slice CT. Gantry allows for a patient port of $70 \mathrm{~cm}$. CT Parameters: It is a single sweep: 120-140 KV and 50-100 mAs (based on body mass index), 0.5 second per CT rotation, Pitch $1.675: 1$, Slice thickness is $5 \mathrm{~mm}$ and $512 \times 512$ matrix. CT acquisition was performed before emission acquisition. CT data were used for image fusion and the generation of the $\mathrm{CT}$ transmission map. No IV contrast was used. Breathing technique is to hold breath after normal expiration. If patient can't do it, then shallow breathing is acceptable.

\section{Image analysis}

The scanned body divided into four regions named head and neck, chest, abdomen, and pelvis. We recorded the body regions of disease involvement in pre-chemotherapy and the first early follow-up post-chemotherapy PET/CT scans. The first early follow-up post-chemotherapy PET/CT scans were reviewed for any residual disease or disease outside the original site seen in the baseline scans. We recorded and listed possible saved body regions if the first early follow-up post-chemotherapy PET/CT scans were limited to the original regions of the disease. Rough estimation of the effective radiation dose in each body region was calculated from the dose length product (DLP) displayed in the dose information display from the CT portion of the $\mathrm{PET} / \mathrm{CT}$ scan using conversion factors in table 1, derived from 2004 CT Quality criteria of the European guidelines for Multislice Computed Tomography (MSCT) (18). Taking on account that radiation dose from low dose CT of the CT portion of the PET/CT is approximately $20 \%$ less than the standard clinical CT dose [19]. Also the possible amount of time could be saved when using PET/CT scan limited to the original disease site was calculated by summing the saved bed PET positions for each body regions excluded in the limited PET/CT scans.

\section{Statistical analysis}

Descriptive statistical analysis was used. Both the possible saved radiation dose (mSv) from CT and scan time (Min) from PET was recorded and listed and the range, average and standard deviation for both of them were calculated. Also the percentage of saved radiation dose and time in 
Table 1 Normalized value of effective dose per DLP over various body regions and (standard) patient age.

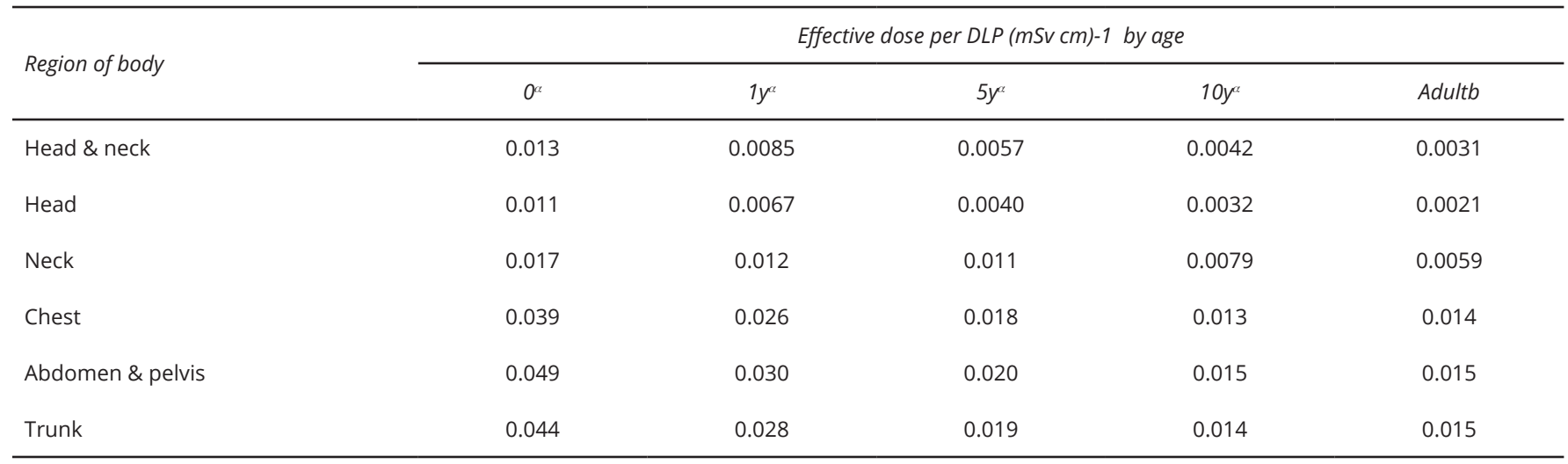

${ }^{\text {aAll }}$ data normalized to $\mathrm{CTDI}$ in the standard head CT dosimetry phantom

${ }^{b}$ Data for head \& neck regions normalized to $\mathrm{CTDI}_{\mathrm{w}}$ in the standard body CT dosimetry phantom

the limited PET/CT scan from the early post chemotherapy Whole body PET/CT scan compared to the initial scan was calculated.

\section{Results}

The study included 100 lymphoma patients (51 male and 49 female with mean age is 38.5 \pm 18.45 years), 56 Hodgkin's Lymphoma (HL), 44 Non-HL. 42 (95.4\%) of the NHL are of diffuse large B-cell lymphoma (DLBCL), one (2.3\%) was T-cell anaplastic large cell lymphoma and one (2.3\%) was B-cell lymphoblastic lymphoma. Thirty nine (88.6\%) of the $\mathrm{NHL}$ are nodal and 5 (11.4\%) are extra nodal (3 thyroid, one gastric and one tonsillar lymphoma). Eighty nine patients received chemotherapy alone and 11 received chemotherapy and radiotherapy (Table 2).

45 out of 100 first post-chemotherapy PET/CT scans showed no significant residual FDG-uptake indicating complete metabolic response (Figure 1), 39 (86.7\%) of them were Hodgkin's disease and 6 (13.3\%) were NHL. Significant residual FDG-uptake (more than liver uptake) at known disease sites was seen in 55 out of 100 first postchemotherapy PET/CT scans indicating residual disease (Figure 2). Out of these 55 scans 30 (55\%) showed disease confined to only one body part, in 22 (40\%) disease was confined to two body parts and in 3 patients (5\%) disease was confined to three body parts. Seventeen (31\%) patients out of 55 with residual disease were $\mathrm{HL}, 8(47 \%)$ of them had disease confined to only one body parts, 5 (29.5\%) patients had disease confined to two body parts and 4 (23.5\%) with disease confined to three body parts. Thirty eight (69\%) patients out of 55 with residual disease were $\mathrm{NHL}, 11(29 \%)$ of them had disease confined to only one body parts, $17(44.7 \%)$ with disease confined to two body parts and $10(26.3 \%)$ with disease confined to three body parts. There is no detected significant FDG uptake in any new site in the first post-chemotherapy PET/CT indicating no unexpected sites of lymphoma (Table 3).

The whole body radiation dose from CT portion of the first post chemotherapy PET/CT scan ranged from $4 \mathrm{mSv}$ to $10 \mathrm{mSv}$ depending on patient's age and number of bed
Table 2 Demographic data and type of lymphoma and therapy of 100 lymphoma patients.

\begin{tabular}{ll}
\hline Characteristics & No. of patients \\
\hline Age & $38.5 \pm 18.45$ years \\
Gender & 51 male and 49 female \\
Type of lymphoma & $56 \mathrm{HL}$ \\
& $44 \mathrm{NHL}$ \\
Histological subtype & $42 \mathrm{DLBCL}$ \\
of NHL & $1 \mathrm{~T}$-cell anaplastic large cell lymphoma \\
& $1 \mathrm{~B}-$ cell lymphoblastic lymphoma \\
& -39 nodal \\
Origin of NHL & -5 extra nodal \\
& $* 3$ thyroid \\
& * 1 gastric \\
& *1 tonsil \\
& 89 chemotherapy \\
Treatment & 11 chemotherapy and radiotherapy \\
\hline
\end{tabular}

positions imaged. When using first post chemotherapy PET/CT scan limited to the sites of known disease, the scan time was reduced by $1(7 \%)$ to $7(50 \%)$ Min (average 5.3 $(38 \%) \pm 1.47 \mathrm{Min})$ as compared to an average of 14 Min scan. Also when first post chemotherapy PET/CT scan was limited to the sites of known disease, the radiation dose (which is roughly estimated from the effective radiation dose in each body region and the DLP using conversion factors) was reduced by $0.7 \mathrm{mSv}(9 \%)$ to $5.9 \mathrm{mSv}(74 \%)$

Table 3 Results of 100 lymphoma patients from first post-chemotherapy PET/CT scans.

\begin{tabular}{lc}
\hline Response to therapy & Number of patients \\
\hline Complete metabolic response & 45 \\
& $39 \mathrm{HL}$ \\
Residual disease & $65 \mathrm{NHL}$ \\
& $17 \mathrm{HL}$ \\
& $38 \mathrm{NHL}$ \\
Patients with residual disease & 19 patients \\
In one body region & $8 \mathrm{HL}$ \\
& $11 \mathrm{NL}$ \\
In two body regions & 22 patients \\
& $5 \mathrm{HL}$ \\
In three body regions & $17 \mathrm{NHL}$ \\
& 14 patients \\
& $4 \mathrm{HL}$ \\
& $10 \mathrm{NHL}$ \\
\hline
\end{tabular}




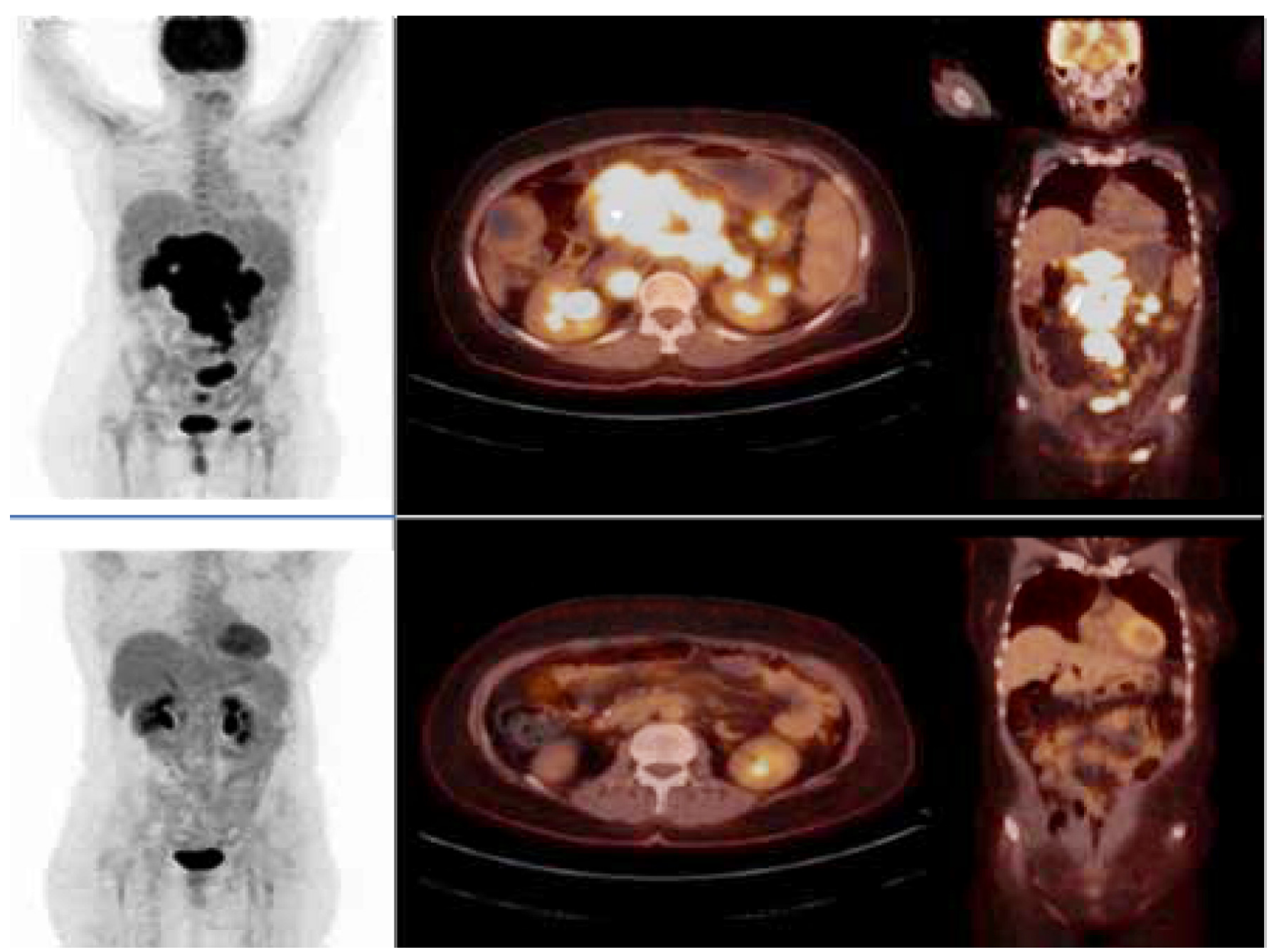

Figure 1 Case of abdomen and pelvic non-Hodgkin's lymphoma: baseline FDG PET/CT images, (A) metabolically active abdominopelvic lymphadenopathy, (B) post chemotherapy treatment FDG PET/CT images, showing complete metabolic response with no disease outside the site of the primary disease.

(average $4.2(52.5 \%) \pm 1.2 \mathrm{mSv}$ ) as compared to an average radiation dose from low dose CT of PET/CT scan of $\sim 8 \mathrm{mSv}$. The supposed reduction of scan time and radiation dose using first post chemotherapy PET/CT scan limited to the sites of known disease, could be accomplished without missing any significant findings.

\section{Discussion}

Evaluation of chemotherapy response is an important aspect for the management of lymphoma patients. 18FFDG PET-CT has an approved role in assessment of response to chemotherapy in lymphoma patients [20]. As early as 1- 3 days after the initiation of therapy changes in FDG uptake have been reported, and the patient's overall disease status can be accurately assessed by the SUV values at 42 days after treatment [2].

According to the ALARA 'as low as reasonably achievable' principle the radiation dose from cancer imaging should be as low as reasonably achievable, though keeping the maximum effectiveness of diagnostic imaging.

Although there are approved significant benefits from the use of integrated PET and CT into routine oncological imaging this comes at the expense of increased radiation dose to the patient compared to either PET or CT alone. As there is a major concern for repeated exposure to ionizing radiation from imaging during management of lymphoma patients which can later lead to life threatening secondary cancers especially in pediatric subjects [21] there is currently a general trend towards decreasing radiation dose for the patients from FDG PET/CT imaging procedure without losing any significant information by previous mentioned multiple methods [11-17].

In this study we investigate the feasibility to reduce scan time and radiation dose by using limited field of view early post therapy FDG PET/CT scan in HL and NHL patients, with imaging confined only to the region of the initial disease site as detected in base line scans. Our study included 100 lymphoma patients, 56 Hodgkin's Lymphoma (HL) and 44 Non-HL of whom 89 patients had chemotherapy alone and 11 patients had chemotherapy and radiotherapy. All of these 100 lymphoma patients have baseline and early post chemotherapy FDG PET/CT scans and have primary disease involved at maximum three body regions. Forty five out100 patients have first post-chemotherapy PET/CT scans with no significant residual FDG-uptake indicating complete metabolic response. Significant residual FDGuptake at known disease sites was seen in 55 out of the 


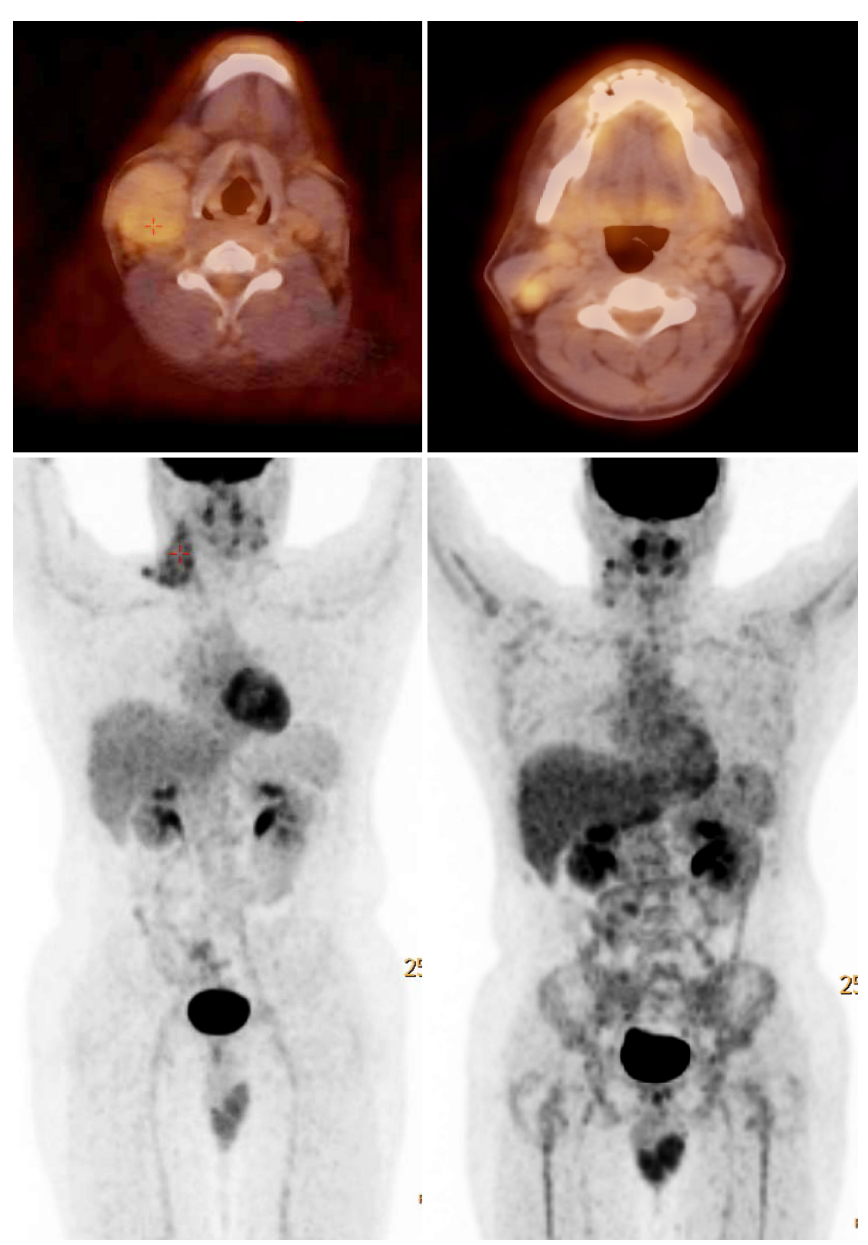

Figure 2 Case of Hodgkin's lymphoma: (A) baseline MIP image and axial neck PET/CT, (B) interim MIP and axial neck PET/CT post 4 cycles of chemotherapy, showing residual lesions in the right cervical region with no disease outside the site of the primary disease.

100 patients in first post-chemotherapy PET/CT scans, indicating residual disease. No patients have new sites of disease in the first post-chemotherapy PET/CT. In our study there is reduction of radiation dose by $0.7(9 \%)$ to $5.9(74 \%) \mathrm{mSv}$ (average $4.2(52.5 \%) \pm 1.2 \mathrm{mSv}$ ) while time was reduced by $1(7 \%)$ to 7 (50\%) Min (average $5.3(38 \%) \pm$ 1.47 Min).

Our results are matched with the results of Sanghera et al. [21], which included 100 lymphoma patients who were assessed pre-chemotherapy with diagnostic CT and post chemotherapy FDG PET/CT. They compared the radiation dose from actual whole body post chemotherapy PET/CT scan and hypothetical limited scan to the disease sites seen in pre chemotherapy CT scan and found $47.9 \%$ mean dose saving and also mean saving of 16 Min per patient when using limited PET/CT scan. The difference between his mean time saving of $16 \mathrm{Min}$ and ours 5.3 Min is due to use of different scanner. The average time per scan using our 16 CT slices TOF PET/CT Philips scanner is $\sim 16$ Min per patient in contrast to $\sim 30$ Min per patient in the over mentioned study using a Discovery ST GE PET/CT with a four-detector spiral CT.

Our results matched with the results of Sonoda et al. [22] as well, who retrospectively analyzed 1000 consecutive lymphoma patients (553 male, mean 42.4-years-old, 421 Hodgkin's Lymphoma (HL), 579 non-HL) and record sites of disease in four conventional body parts (head-and-neck, thorax, abdomen and pelvis) on pre-chemotherapy PET/ CT and the first interim post-chemotherapy. His results showed that no FDG-uptake was seen in 744/1000 first post-chemotherapy PET/CT, FDG uptake at known disease sites was seen in 256/1000 cases, 902/1000 of the patients had disease confined to only one or two body parts out of four. Post-chemotherapy PET/CT did not reveal any unexpected sites of lymphoma or incidental interval malignancy. Limiting PET/CT to the sites of known disease would have reduced a mean radiation dose by $3.6 \mathrm{mSv}$ (24.3\%), with a mean time-saving of 16 Min (66.6\%).

\section{Limitation of the study}

The limitation of our study includes less number of studied patients and use of rough estimation for radiation dose from PET/CT. Moreover the short period of follow-up prior to the first post chemotherapy PET/CT scan renders the results of this study only applicable to short term follow up post therapy but likely not to long term follow up post therapy to detect disease recurrence or restaging.

\section{Further analysis}

We intended to continue our study to include large number of patients. Also it would be helpful to use dedicated software to calculate more accurately the radiation dose from $C T$ portion of $P E T / C T$ for individual scans like IMPACT software. We might extend the follow up period post therapy to assess the tendency of developing lesions outside the primary sites of disease detected in the baseline scan.

\section{Conclusion}

It is not mandatory to do WB 18F-FDG PET/CT scan in assessment of early response to chemotherapy in curable lymphoma and it may be sufficient to limit the scan to the sites of initial disease without missing any significant findings, resulting in significantly reducing the total radiation dose and time saving. Further studies with larger patients' population may be considered for confirmation of the current results.

\section{Conflict of interest}

The authors declare no conflict of interest.

\section{References}

[1] Hutchings M. PET imaging in lymphoma. Expert Rev Hematol. 2009; 2(3):261-276.

[2] Halanaik D, Rakesh K. F-18 FDG PET/PET/CT in the Management of Lymphoma. Indian journal of medical \& paediatric oncology. 2007; 28(3):17-23.

[3] The Royal College of Physician, Royal college of Radiologists. Evidencebased indications for the use of PET/CT in the UK 2013. London: RCP, RCR, Royal College of Physicians 2013.

[4] Delbeke D, Coleman RE, Guiberteau MJ, Brown ML, Royal HD, et al Procedure guideline for tumor imaging with 18F-FDG PET/CT 1.0.J Nucl Med. 2006; 47(5):885-895.

[5] Esma AA, Drew AT. Considerations Regarding Radiation Exposure in Performing FDG-PET/CT. American College of Radiology 2012; 1-7. 
[6] Willowson KP, Bailey EA, Bailey DL. A retrospective evaluation of radiation dose associated with low dose FDG protocols in whole-body PET/CT. Australas Phys Eng Sci Med. 2012; 35(1):49-53.

[7] Brix G, Lechel U, Glatting G, Ziegler SI, Münzing W, et al. Radiation exposure of patients undergoing whole-body dual-modality $18 \mathrm{~F}-\mathrm{FDG}$ PET/CT examinations. J Nucl Med. 2005; 46(4):608-613.

[8] Huang B, Law MW, Khong PL. Whole-body PET/CT scanning: estimation of radiation dose and cancer risk. Radiology. 2009; 251(1):166-174.

[9] Wu TH, Huang YH, Lee JJ, Wang SY, Wang SC, et al. Radiation exposure during transmission measurements: comparison between CT- and germanium-based techniques with a current PET scanner. Eur J Nucl Med Mol Imaging. 2004; 31(1):38-43.

[10] Khamwan K, Krisanachinda A, Pasawang P. The determination of patient dose from (18)F-FDG PET/CT examination. Radiat Prot Dosimetry. 2010; 141(1):50-55.

[11] Murray I, Kalemis A, Glennon J, Hasan S, Quraishi S, et al. Time-of-flight $\mathrm{PET} / \mathrm{CT}$ using low-activity protocols: potential implications for cancer therapy monitoring. Eur J Nucl Med Mol Imaging. 2010; 37(9):16431653.

[12] Conti M. Focus on time-of-flight PET: the benefits of improved time resolution. Eur J Nucl Med Mol Imaging. 2011; 38(6):1147-1157.

[13] Boellaard R, O'Doherty MJ, Weber WA, Mottaghy FM, Lonsdale MN, et al. FDG PET and PET/CT: EANM procedure guidelines for tumor PET imaging: version 1.0. Eur J Nucl Med Mol Imaging. 2010; 37(1):181200.

[14] Jackson J, Pan T, Tonkopi E, Swanston N, Macapinlac HA, et al. Implementation of automated tube current modulation in PET/ CT: prospective selection of a noise index and retrospective patient analysis to ensure image quality. J Nucl Med Technol. 2011; 39(2):8390.

[15] Farghaly $\mathrm{H}$, Kassem $\mathrm{H}$, Alobaid H. FDG-PET/CT in management of head and neck cancers: Is it mandatory to do Whole Body Scan Egyptian J Nucl Med. 2012; 5(1).

[16] Huang YT, Ravi AS. Potential for truncating the scan length of restaging FDG-PET/CT after chemoradiotherapy in head and neck squamous cell carcinoma. Nucl Med Commun. 2012; 33(5):503-508.

[17] Jamar F, Buscombe J, Chiti A, Christian PE, Delbeke D, et al. EANM/ SNMMI guideline for 18F-FDG use in inflammation and infection. J Nucl Med. 2013; 54(4):647-658.

[18] Bongartz G, Golding SJ, Jurik, AG, Calzado A, Geleijns J, et al. European Guidelines for Multislice Computed Tomography, CT Quality Criteria 2004; 1-107.

[19] Environmental Health \& Safety. Dose Estimates for Nuclear Medicine Scans, Columbia University, Available on February 2013 from: http:// www.ehs.columbia.edu/Dosimetry

[20] Guay C, Lepine M, Verreault J, Bénard F. Prognostic value of PET using 18F-FDG in Hodgkin's disease for posttreatment evaluation. J Nucl Med. 2003; 44(8):1225-1231.

[21] Kumar R, Shandal V, Shamim S, Halanaik D, Malhotra A. Clinical applications of PET and PET/CT in pediatric malignancies. Expert Rev Anticancer Ther. 2010; 10(5):755-768.

[22] Sanghera B, Sonoda LI, Hart J, Vivian G, Mills T, et al. Age and doselimited PET-CT scan regime in lymphoma: between the devil and the deep blue sea? Radiat Prot Dosimetry. (2011); 150(3):381-384.

[23] Luke S, Subhadip GR, Mahrukh Q. A new radiation dose minimization protocol in early chemotherapy response in lymphoma using 18F-FDG PET/CT. J Nucl Med. 2013; 54(Supplement 2):177. 